\title{
Cuidados de enfermería en el paciente con enfermedad renal crónica en hemodiálisis: una revisión sistemática
}

\author{
Zahira Esperanza Ángel Ángel, Germán Alberto Duque Castaño, David Leonardo Tovar Cortes \\ Fundación Universitaria de Ciencias de la Salud - Fucs. Colombia
}

\section{Resumen}

Introducción: La enfermedad renal crónica es considerada actualmente un problema de salud pública en el ámbito mundial por su prevalencia e incidencia creciente en la población, su importancia relativa en la carga de enfermedad del país, su comportamiento crónico o permanente, su potencial letal, y su impacto en el paciente, su familia y los sistemas sanitarios. Por lo tanto requiere una atención integral fundamentada en buenas prácticas clínicas basadas en la evidencia.

Método: Revisión sistemática de la literatura científica de artículos publicados entre 2004 a 2014, en idioma español, portugués e inglés, y accesibles a texto completo. Búsqueda exhaustiva y reproducible de trabajos originales en las bases de datos Cuiden Plus, CINAHL, SciELO, Lilacs, PubMed, y Nursing Ovid. Valoración crítica de la calidad científica de los estudios aplicando las parrillas de investigación de CASPe, y la lista de chequeo STROBE.

Resultados: Los temas que surgieron del análisis de los artículos fueron: Perspectiva sobre modelos y teorías de enfermería en el cuidado del paciente en hemodiálisis, lenguaje común enfermero, diagnósticos de enfermería en el paciente en hemodiálisis, educación y autocuidado del paciente en hemodiálisis, importancia de la familia en el cuidado del paciente, satisfacción del paciente como objetivo de calidad.

Conclusiones: Los modelos, teorías y taxonomía permiten una visión más amplia, profunda y abarcadora de

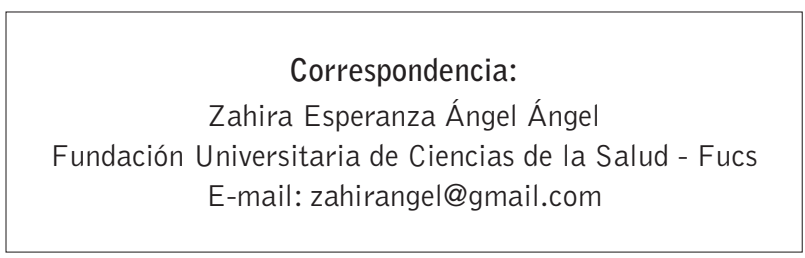

la enfermería que viabiliza el camino a la excelencia en la atención al paciente.

PALABRAS CLAVE

- ATENCIÓN DE ENFERMERÍA

- INSUFICIENCIA RENAL CRÓNICA

- DIÁLISIS RENAL

- PROCESOS DE ENFERMERÍA

- PLANIFICACIÓN DE ATENCIÓN AL PACIENTE

- CUIDADO DE ENFERMERÍA

Nursing care plans for chronic kidney disease patients in hemodialysis: a systematic review

\section{Abstract}

Introduction: Chronic Kidney Disease (CKD) is considered a current public health problem worldwide due to its prevalence and growing incidence in the population, its relative importance in the health burden of our country, its permanent chronic behavior, its lethal potential and its impact on the patient, family and nations. Therefore requires a comprehensive care based on good clinical practice based on evidence.

Method: A systematic scientific literature review of the articles published between 2004 and 2014, done in Spanish, Portuguese and English, and available in full text. A thorough search for original works in databases such as Cuiden Plus, CINAHL, SciELO, Lilacs, PubMed, and Nursing Ovid. A critical assessment of the scientific quality of the studies, applying the CASPe research grids, and the STROBE checklist.

Findings: The themes that came out as a result of the articles analysis were: Perspective on Nursing models 
and theories for caring patients in hemodialysis; Common nursing language and nursing care process; Nursing diagnosis for the patient in hemodialysis; Education for the patient in hemodialysis; Self-care for the patient in hemodialysis; Family's important role when caring for a patient; Patient satisfaction as a quality objective. Conclusions: Nursing models, theories and taxonomy allow a broader, deeper and comprehensive view of nursing that eases the path to excellence in patient care.

\section{KEYWORDS}

- NURSING CARE

- CHRONIC KIDNEY DISEASE

- KIDNEY DIALYSIS

- NURSING PROCESS

- NURSING CARE PLANS

\section{Introducción}

La Enfermedad Renal Crónica (ERC) es considerada actualmente un problema de salud pública en el ámbito mundial por su prevalencia e incidencia creciente en la población, su importancia relativa en la carga de enfermedad del país, su comportamiento crónico o permanente, su potencial letal y porque representa un importante gasto en salud para el sistema, dado que requiere una alta complejidad técnica en su manejo ${ }^{1}$. En Colombia más de 25 mil pacientes se encuentran en estadio 5 (grado más avanzado de la enfermedad) requiriendo terapias de reemplazo renal, siendo la diálisis el procedimiento de alto costo de mayor frecuencia en esta población ${ }^{2}$.

Además del gran impacto socioeconómico que producen las enfermedades crónicas, también limitan o ponen en riesgo la existencia y la calidad de vida de quien las tiene, $y$ de todo el sistema de relaciones interpersonales en que está implicada la persona y su familia, que ve seriamente afectada toda su dinámica y funcionamiento ${ }^{3,4}$. En este sentido la ERC debe ser considerada como una condición que requiere un ambiente humanístico para su cuidado ${ }^{5}$, así como un abordaje integral encaminado a un diálogo y trabajo colaborativo entre los profesionales que brindan sus cuidados al individuo con la enfermedad y su familia. De tal forma, es primordial reconocer el papel que desempeña enfermería como disciplina que lidera el cuidado, y como principal responsable en la monitorización y control de la asistencia sanitaria prestada a los pacientes cada día en diversos entornos ${ }^{6}$.
Con el fin de brindar la mejor calidad de cuidados de enfermería a los usuarios que los requieren, las/los enfermeras/os han creado el Proceso de Atención de Enfermería (PAE) como un método de trabajo que aporta grandes beneficios, al lograr la vinculación del paciente a su cuidado, como ente participativo del mismo; de igual forma el PAE ha proporcionado ganancia profesional, pues al trabajar con un método estructurado, posibilita la unificación de criterios entre enfermeros, todo esto orientado hacia el desarrollo y crecimiento de la profesión de enfermería?.

Al utilizar el PAE con la taxonomía diagnóstica de la NANDA, en la atención de pacientes con insuficiencia renal en hemodiálisis, proporciona las bases para la obtención de las intervenciones de enfermería más eficaces para llegar a unos resultados óptimos de cuidado; igualmente la utilización de los diagnósticos enfermeros favorece un lenguaje común y coherente, que representa el definir el cuerpo de conocimientos de enfermería, por la autonomía profesional que les otorga y por la continuidad de participación y alto nivel de calidad en los cuidados que recibe el usuario ${ }^{8-10}$. Lo que aporta una evidencia y refleja la eficacia de la labor de enfermería en el funcionamiento de la unidad de hemodiálisis, garantizando la atención integral al paciente, la continuidad de cuidados y el seguimiento diario, trasmitiendo seguridad y confianza a este, tanto en su relación con los profesionales sanitarios como en la aceptación de su enfermedad ${ }^{11-17}$.

Es por ello que se considera importante la investigación basada en la evidencia, con el fin de revisar juiciosamente la mejor evidencia científica clínica disponible para tomar decisiones sobre el cuidado de cada paciente ${ }^{18}$; y de esta manera buscar constantemente la integración de enfoques, perspectivas y puntos de vista de lo que es la práctica profesional y la investigación, con la tradición científica de la enfermería y la experiencia acumulada en el manejo de modelos de práctica y modelos teóricos ${ }^{19}$.

Es así como surge este trabajo con el propósito de revisar las evidencias científicas que proporcionan elementos para generar planes de cuidado que orienten la práctica de enfermería en la atención del paciente con enfermedad renal crónica en hemodiálisis.

\section{Metodología}

Se realizó una revisión sistemática, en 7 fases de acuerdo a lo planteado por Guerra, Muñoz y Santos ${ }^{20}$; se llevó a cabo una búsqueda exhaustiva y reproducible en las 
bases de datos Cuiden Plus, CINAHL, SciEL0, Lilacs, PubMed, y Nursing Ovid, con los descriptores MeSH (DeCS): Nursing Care, Renal Insufficiency Chronic, Renal Dialysis, Nursing Process, Patient Care Planning y sus equivalentes en español y portugués. Se diseñaron ecuaciones de búsqueda con los operadores booleanos AND y OR, la búsqueda se realizó en los meses de agosto a diciembre de 2014, desde los servidores de la Fundación Universitaria de Ciencias de la Salud (FUCS).

Los criterios de inclusión fueron: artículos de investigación cuantitativa y cualitativa publicados en los últimos 10 años (2004-2014), en idiomas español, portugués e inglés, y accesibles a texto completo.

Selección y recuperación de los estudios que cumplen los criterios: Se seleccionaron 56 artículos inicialmente, luego se excluyeron 31 artículos que no cumplían con los criterios, quedando 25 artículos, los cuales fueron sometidos a lectura crítica con las herramientas de $\mathrm{CASPe}^{21} \mathrm{y}$ STROBE 22 de acuerdo al tipo de estudio, de los cuales se seleccionaron finalmente 21 artículos. Figura 1

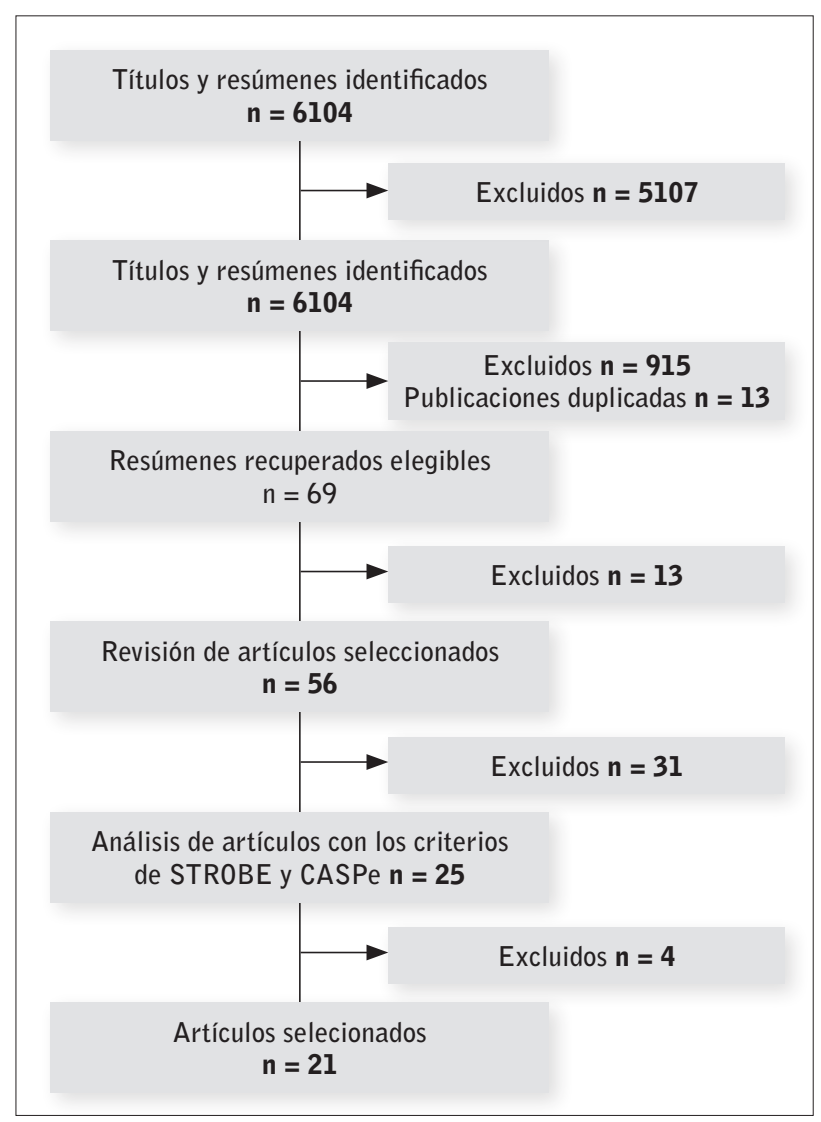

Figura 1. Flujograma del proceso de selección de artículos.

Fuente: Ángel, Z. Duque, G. y Tovar, L. Cuidados de enfermería en el paciente con Enfermedad Renal Crónica en Hemodiálisis: Una revisión sistemática. 2015.
Se organizó la información en una base de datos de Microsoft Excel ${ }^{\circledR}$. Tres evaluadores asignaron la puntuación de los artículos después de realizar la lectura crítica de estos, con los siguientes valores 0 : si no cumplía los criterios de selección; 1: no se sabía si cumplía con los criterios de selección; 2: si cumplía con los criterios de selección. Se analizó el promedio y la desviación estándar en donde los artículos que cumplieron los criterios de selección tenían un promedio igual o mayor a 1,54. Tabla 1.

Consideraciones éticas: En esta investigación se reconoce la propiedad intelectual, la generación y aplicación del conocimiento, lo que implica dar fe de los estudios que permitieron y soportaron el marco de antecedentes, el marco teórico y el diseño metodológico, en las referencias bibliográficas.

\section{Resultados}

Se seleccionaron 17 artículos de investigación cualitativa y 4 artículos de investigación cuantitativa, para un total de 21 artículos seleccionados. El país que reporta mayor número de investigaciones de la temática en estudio es Brasil.

Los temas que surgieron del análisis de los artículos fueron: perspectiva sobre modelos y teorías de enfermería en el cuidado del paciente en hemodiálisis, lenguaje común enfermero y proceso de atención de enfermería, diagnósticos de enfermería en el paciente en hemodiálisis, educación en el paciente en hemodiálisis, autocuidado del paciente en hemodiálisis, importancia de la familia en el cuidado del paciente, y satisfacción del paciente como objetivo de calidad.

\section{Análisis de los resultados. \\ Perspectiva sobre modelos y teorías de enfermería en el cuidado del paciente en hemodiálisis}

Brindarle cuidado a un paciente con enfermedad renal crónica requiere una preparación especial de las personas que los cuidan, y posee un grado de complejidad mayor que otros campos de la enfermería, requiere estar atento a integrar todos los conocimientos y habilidades en pro del bienestar del paciente. No obstante la rutina diaria de usar una máquina para atender a estos pacientes en hemodiálisis puede llevar a perder el verdadero sentido de la profesión de enfermería que va 
Tabla 1. Promedio y desviación estándar de los artículos seleccionados.

\section{ARTÍCULOS}

EVDOR \#1 EVDOR \#2 EVDOR \#3 PROM

1. Professional support for next of kin of patients receiving chronic hemodialysis treatment: a content analysis study of nursing documentation.

2. Cuidado de enfermería para clientela en hemodiálisis: sus dimensiones instrumentales y expresivas

3. Providing and receiving nursing care during hemodialysis 1,8

4. Análisis de los factores que influyen en la aparición de complicaciones y supervivencia de los catéteres venosos centrales para hemodiálisis

5. Effect of health contract intervention on renal dialysis patients in Korea

6. Diagnósticos de enfermagem em pacientes renais crônicos em hemodiálise

7. Diagnósticos de enfermagem em adultos em tratamento de hemodiálise

8. Diagnósticos de enfermagem identificados em pacientes hospitalizados durante sessões de hemodiálise

9. Diagnósticos de enfermería en pacientes sometidos a hemodiálisis

10. Diagnósticos de enfermagem em pacientes submetidos à hemodiálise: semelhanças entre o modelo de adaptação e a NANDA internacional

11. Profile of nursing diagnoses before starting hemodialitic treatment

12. Componentes Do Modelo Teórico De Roy Em Pacientes Submetidos À Hemodiálise

13. Perspectiva sobre modelos y teorías de enfermería en el ámbito de nefrología

14. Satisfacción del paciente en una unidad de hemodiálisis: Objetivo de calidad asistencial en enfermería

1,8

1,9

(2)

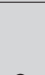

1,9

1,5

15. Prevalence of nursing diagnosis of fluid volume excess in patients undergoinghemodialysis

\begin{tabular}{|c|c|c|c|}
\hline 1,7 & 1,5 & 1,6 & 1,6 \\
\hline 1,7 & 1,8 & 1,8 & 1,76 \\
\hline 1,5 & 1,6 & 1,7 & 1,6 \\
\hline 1,4 & 1,7 & 1,6 & 1,56 \\
\hline 1,5 & 1,4 & 1,6 & 1,5 \\
\hline
\end{tabular}

1,6

\begin{tabular}{l|l|l|l|}
1,6 & 1,8 & 1,6 & 1,66 \\
\hline 1,5 & 1,5 & 1,7 & 1,56 \\
\hline
\end{tabular}

1,5

16. Nursing diagnosis ineffective protection: content validation in patients underhemodialysis.

1,8

1,8

1,7

\begin{tabular}{c|c|c|c}
1,8 & 2 & 1,7 & 1,83 \\
\hline 1,7 & 1,7 & 1,4 & 1,6 \\
\hline 1,7 & 2 & 1,5 & 1,73 \\
\hline 1,6 & 1,4 & 1,5 & 1,5 \\
\hline
\end{tabular}

17. Evaluación de la opinión de los pacientes con enfermedad renal crónica en diálisis respecto al fin de la vida y la planificación anticipada de cuidados

\begin{tabular}{cccc}
1,6 & 1,9 & 1,5 & 1,66 \\
\hline 1,6 & 1,9 & 1,8 & 1,76 \\
\hline 1,7 & 1,7 & 1,8 & 1,73 \\
\hline
\end{tabular}

\begin{tabular}{|c|c|c|c|c|}
\hline anticipada de cuidados & 1,6 & 1,7 & 1.5 & 1,65 \\
\hline $\begin{array}{l}\text { 18. Necessidades de orientação de enfermagem } \\
\text { para o autocuidado de clientes em terapia de hemodiálise }\end{array}$ & 1,6 & 1,5 & 1.5 & 1,55 \\
\hline $\begin{array}{l}\text { 19. Aplicación de una técnica cualitativa para la elaboración de un plan } \\
\text { de atención al paciente pluripatológico en hemodiálisis }\end{array}$ & 1,5 & 1,5 & 1,5 & 1,5 \\
\hline $\begin{array}{l}\text { 20. Utilização de metodologia ativa no ensino e assistência de enfermagem } \\
\text { na produção nacional: revisão integrativa }\end{array}$ & 2 & 1,9 & 1,9 & 1,93 \\
\hline $\begin{array}{l}\text { 21. Sistemas de organización de cuidados en enfermería: reflexiones sobre } \\
\text { la complejidad del cuidado como práctica asistencial }\end{array}$ & 1,5 & 2 & 2 & 1,83 \\
\hline PROMEDIO & & & & 1,66 \\
\hline DESVIACIÓN ESTÁNDAR & & & & 0,12 \\
\hline TOTAL & & & & 1,54 \\
\hline
\end{tabular}

Fuente: Ángel, Z. Duque, G. y Tovar, L. Cuidados de enfermería en el paciente con Enfermedad Renal Crónica en Hemodiálisis: Una revisión sistemática. 2015. 
Tabla 2. Nivel de evidencia y grado de recomendación de artículos.

\begin{tabular}{|c|c|c|}
\hline ARTÍ́CULOS & $\begin{array}{l}\text { NIVEL DE } \\
\text { EVIDENCIA }\end{array}$ & $\begin{array}{l}\text { GRADO DE } \\
\text { RECOMENDACIÓN }\end{array}$ \\
\hline $\begin{array}{l}\text { 1. Professional support for next of kin of patients receiving chronic hemodialysis treatment: } \\
\text { a content analysis study of nursing documentation. }\end{array}$ & III & B \\
\hline $\begin{array}{l}\text { 2. Cuidado de enfermería para clientela en hemodialisis: } \\
\text { sus dimensiones instrumentales y expresivas }\end{array}$ & II-2 & B \\
\hline 3. Providing and receiving nursing care during hemodialysis & III & B \\
\hline $\begin{array}{l}\text { 4. Análisis de los factores que influyen en la aparición de complicaciones y supervivencia } \\
\text { de los catéteres venosos centrales para hemodiálisis }\end{array}$ & III & A \\
\hline 5. Effect of health contract intervention on renal dialysis patients in Korea & I & A \\
\hline 6. Diagnósticos de enfermagem em pacientes renais crônicos em hemodiálise & II-2 & B \\
\hline 7. Diagnósticos de enfermagem em adultos em tratamento de hemodiálise. & III & B \\
\hline $\begin{array}{l}\text { 8. Diagnósticos de enfermagem identificados em pacientes hospitalizados } \\
\text { durante sessões de hemodiálise }\end{array}$ & II-2 & B \\
\hline 9. Diagnósticos de enfermería en pacientes sometidos a hemodiálisis & III & B \\
\hline $\begin{array}{l}\text { 10. Diagnósticos de enfermagem em pacientes submetidos à hemodiálise: } \\
\text { semelhanças entre o modelo de adaptação e a NANDA internacional }\end{array}$ & II-2 & B \\
\hline 11. Profile of nursing diagnoses before starting hemodialysis treatment & II-3 & B \\
\hline 12. Componentes Do Modelo Teórico De Roy Em Pacientes Submetidos À Hemodiálise & II-2 & B \\
\hline 13. Perspectiva sobre modelos y teorías de enfermería en el ámbito de nefrología & III & B \\
\hline $\begin{array}{l}\text { 14. Satisfacción del paciente en una unidad de hemodiálisis: } \\
\text { Objetivo de calidad asistencial en enfermería }\end{array}$ & III & B \\
\hline 15. Prevalence of nursing diagnosis of fluid volume excess in patients undergoinghemodialysis & II-2 & B \\
\hline 16. Nursing diagnosis ineffective protection: content validation in patients underhemodialysis. & III & B \\
\hline $\begin{array}{l}\text { 17. Evaluación de la opinión de los pacientes con enfermedad renal crónica en diálisis } \\
\text { respecto al fin de la vida y la planificación anticipada de cuidados }\end{array}$ & III & A \\
\hline $\begin{array}{l}\text { 18. Necessidades de orientação de enfermagem } \\
\text { para o autocuidado de clientes em terapia de hemodiálise }\end{array}$ & III & B \\
\hline $\begin{array}{l}\text { 19. Aplicación de una técnica cualitativa para la elaboración de un plan de atención } \\
\text { al paciente pluripatológico en hemodiálisis }\end{array}$ & III & B \\
\hline $\begin{array}{l}\text { 20. Utilização de metodologia ativa no ensino e assistência de enfermagem na produção } \\
\text { nacional: revisão integrativa }\end{array}$ & III & B \\
\hline $\begin{array}{l}\text { 21. Sistemas de organización de cuidados en enfermería: reflexiones sobre la complejidad } \\
\text { del cuidado como práctica asistencial }\end{array}$ & III & C \\
\hline
\end{tabular}

Fuente: Ángel, Z. Duque, G. y Tovar, L. Cuidados de enfermería en el paciente con Enfermedad Renal Crónica en Hemodiálisis: Una revisión sistemática. 2015.

enfocada al cuidado, para ello existen ciertas actividades que involucran a la/el enfermera/o con el paciente teniendo contacto físico, uno de los puntos importantes para recordar que la atención debe ser personalizada, enfocada al bienestar común, y brindada por un profesional23.
De ahí que el cuidado de enfermería requiere integrar todos aquellos conocimientos de la profesión y todas aquellas actitudes de los profesionales para tener un impacto positivo en el bienestar de los pacientes. Es así como los modelos y teorías de enfermería brindan posibilidades de intervención, de acción inmediata, e igual- 
mente brinda al paciente las suficientes herramientas de cuidado.

Sin embargo continúa el reto para enfermería de lograr que los profesionales de esta disciplina integren a su práctica clínica diaria este cuerpo de conocimientos. Algunos de los factores que describe el profesional como obstáculos para su implementación, son: el modelo que usaba como estudiante no le es útil en la labor diaria y los documentos de registros actuales de enfermería no favorecen trabajar bajo un modelo, posiblemente porque han quedado obsoletos; también porque son complejos y no se conocen en profundidad. Al mismo tiempo por el poco apoyo de las instituciones en su implementación, la ausencia de modelos innovadores en un ambiente cambiante, y la poca formación posgradual en modelos que tienen estos profesionales $^{24}$.

De esta forma, se concluye sobre la importancia que tiene la aplicación en la práctica clínica, de los fundamentos filosóficos y teorías de enfermería, pues no solo constituye un instrumento de perfeccionamiento de la atención al paciente sino que permite una visión más amplia, profunda y abarcadora de la enfermería que viabiliza el camino a la excelencia en la atención al paciente ${ }^{25}$. De igual forma se hace fundamental preparar al profesional a nivel de pregrado y posgrado en el conocimiento e implementación de modelos y teorías a su práctica diaria, así como implementar mecanismos en las instituciones para su uso.

\section{Lenguaje enfermero y/o taxonomías}

Un lenguaje común propio del profesional de enfermería aumenta la comunicación entre los profesionales y los pacientes, permite tener claro el objeto de estudio y saber con exactitud qué problemas resuelve el profesional de enfermería y que sean de su total competencia, además permite la sistematización del trabajo y la calidad de los cuidados prestados ${ }^{26}$.

La taxonomía NANDA permite el uso de lenguaje de diagnósticos estandarizados para documentar lo realizado por enfermería y de una forma efectiva lograr un entendimiento interdisciplinar que se integre a la práctica asistencial27-29. Para llegar a la identificación de los diagnósticos de enfermería se ha utilizado el Proceso de Atención de Enfermería, que es un método sistematizado de identificación y resolución de problemas de salud, siempre dirigido a cubrir las necesidades del cliente o del sistema familiar. Consta de cinco fases:
Valoración, Diagnóstico de Enfermería, Planificación, Ejecución y Evaluación ${ }^{26}$.

A pesar de la relevancia del proceso enfermero en el cuidado de estos pacientes y sus familias, se observa que en la mayoría de los estudios revisados solo llegan a la segunda fase del proceso que es la identificación de diagnósticos prioritarios de los pacientes en hemodiálisis. Por lo cual se observa la necesidad de ampliar las investigaciones en las otras fases del proceso de atención de enfermería y poder evaluar de forma más amplia el proceso completo.

\section{Diagnósticos de Enfermería en el paciente en Hemodiálisis}

Determinar los diagnósticos de enfermería comunes en los sujetos sometidos a hemodiálisis, ayuda a los profesionales de enfermería en la atención a los pacientes renales crónicos proporcionando herramientas para la planificación de la asistencia ${ }^{11-14,30}$.

Los autores describen diagnósticos de enfermería reales, los cuales detallan problemas y alteraciones de la salud del individuo, validados clínicamente mediante la presencia de signos y síntomas que lo definen; y diagnósticos de enfermería de riesgo, definidos como los juicios clínicos que realiza la enfermera en los que valora la existencia de una mayor vulnerabilidad de la persona a desarrollar un problema concreto que el resto de la población en la misma situación ${ }^{31}$.

Los diagnósticos de enfermería reales en pacientes en hemodiálisis más frecuentes, descritos en la literatura, son: volumen de líquidos excesivo, eliminación urinaria afectada, perfusión tisular ineficaz: renal, hipotermia, movilidad física disminuida, debilidad, fatiga, intolerancia a la actividad, integridad de la piel afectada, disfunción sexual, deterioro de la dentición, percepción sensorial perturbada (visual y auditiva), padrón de sueño alterado, insomnio, memoria afectada, dolor agudo, dolor crónico, estreñimiento, diarrea, falta de adhesión, conocimientos deficientes, control ineficaz del régimen terapéutico, déficit de autocuidado, desequilibrio en la nutrición por defecto, comportamiento de salud propenso a riesgo, control ineficaz de la salud, temor, ansiedad, desesperanza, y aislamiento social. En cuanto a los diagnósticos potenciales de enfermería más frecuentes en la misma población son: riesgo de desequilibrio electrolítico, riesgo de infección, riesgo de caídas, riesgo de glicemia inestable, riesgo de soledad, y riesgo de baja autoestima situacional ${ }^{12,26-29,32-34}$. Ver Tabla 3. 
Tabla 3. Diagnósticos de Enfermería más frecuentes en el paciente con Enfermedad Renal Crónica en hemodiálisis.

\begin{tabular}{|c|l|l|l}
\hline$N^{\circ}$ & DIAGNósticos REALES DE ENFERMERÍA & N $^{\circ}$ & DIAGNóSTICOS REALES DE ENFERMERÍA \\
\hline 1 & Volumen de líquidos excesivo & 15 & Memoria afectada \\
\hline 2 & Eliminación urinaria afectada & 16 & Dolor agudo \\
\hline 3 & Perfusión renal ineficaz & 17 & Dolor crónico \\
\hline 4 & Hipotermia & 18 & Estreñimiento \\
\hline 5 & Movilidad física disminuida & 19 & Diarrea \\
\hline 6 & Debilidad - Fatiga & 20 & Falta de adhesión \\
\hline 7 & Intolerancia a la actividad & 21 & Conocimientos deficientes \\
\hline 8 & Integridad de la piel afectada & 22 & control ineficaz del régimen terapéutico \\
\hline 9 & Disfunción sexual & 23 & Déficit de autocuidado \\
\hline 10 & Deterioro de la Dentición & 24 & Desequilibrio en la nutrición por defecto \\
\hline 11 & Percepción sensorial perturbada (visual y auditiva) & 25 & Comportamiento de salud propenso a riesgo \\
\hline 12 & Padrón de sueño alterado & 26 & Control ineficaz de la salud \\
\hline 13 & Insomnio & 27 & Desesperanza \\
\hline 14 & Temor y Ansiedad & 28 & Aislamiento social \\
\hline & & & \\
\hline
\end{tabular}

\begin{tabular}{l|l|l|l} 
& & & DIAGNósticos POTENCIALES \\
\hline 25 & Riesgo de desequilibrio electrolítico & 28 & Riesgo de glicemia inestable \\
\hline 26 & Riesgo de infección & 29 & Riesgo de baja autoestima situacional \\
\hline 27 & Riesgo de caídas & 30 & Riesgo de soledad \\
\hline
\end{tabular}

Fuente: Ángel, Z. Duque, G. y Tovar, L. Cuidados de enfermería en el paciente con Enfermedad Renal Crónica en Hemodiálisis: Una revisión sistemática. 2015.

Un diagnóstico real identificado por todos los autores es el exceso de líquido en los pacientes con la enfermedad renal en hemodiálisis, pues este conduce a complicaciones, tanto como hipotensión y calambres debido a la retirada de líquidos y electrolitos, así como trastornos cardiovasculares que pueden ser graves e irreversibles. Los estudios muestran que el consumo de alimentos ricos en sodio, así como la ingesta excesiva de líquidos, y la disminución de la diuresis residual están directamente relacionados al aumento de peso y aumento de la presión arterial pre-diálisis. Aunque el tratamiento de hemodiálisis puede ser eficaz, no sustituye completamente la función renal, de modo que la retención de agua sigue siendo una de los principales problemas identificados en estos pacientes $^{27,28,35-38}$.

Así mismo, el diagnóstico de hipotermia se detectó como una complicación potencial durante las sesiones de diálisis. Se sabe que la baja temperatura se presenta en pacientes sometidos a hemodiálisis por enfriamiento de la sangre por la circulación extracorpórea, ya que la línea de sangre y/o solución de dializado están expuestos a la temperatura ambiente, lo que provoca la pérdida de calor por convección, por tal razón enfermería debe ser consciente de esta situación para evitar dicha complicación y proporcionar comodidad al paciente ${ }^{27-29}$.

Por otro lado, se presentan los diagnósticos de debilidad, fatiga e intolerancia a la actividad, concomitantes a condiciones de anemia, como una complicación de la enfermedad renal crónica, con diversas causas, así como la deficiencia relativa de eritropoyetina y la deficiencia de hierro. La anemia causa palidez, debilidad, malestar, déficit de atención, deterioro de la calidad de vida y mayor mortalidad en pacientes con enfermedad renal crónica, la evidencia indica que la anemia acelera la disminución de la función renal y su corrección puede interferir favorablemente en la evolución de la ERC. Por lo tanto, el personal de enfermería debe tener el objetivo de la corrección de la anemia, y así reducir la necesidad de transfusiones y el número hospitalizaciones, mejorando la calidad de vida, la capacidad cognitiva y el rendimiento físico en las actividades diarias ${ }^{29.38}$. 
El diagnóstico de déficit de autocuidado, comprendido como una falta o una capacidad no adecuada del individuo para realizar todas las actividades necesarias que garanticen un funcionamiento (pro) saludable, se encuentra relacionado con los diagnósticos de conocimientos deficientes, control ineficaz del régimen terapéutico, control ineficaz de la salud, y falta de adhesión, los cuales al identificarse permite a enfermería analizar las causas de ese déficit, dadas por la condición del paciente, los factores biopsicosociales y culturales del paciente y su familia, los sistemas de soporte, las necesidades de aprendizaje y las estrategias que está utilizando para afrontar su situación ${ }^{37}$, las cuales pueden estar relacionadas con diagnósticos como temor, ansiedad, y desesperanza.

Finalmente, en cuanto a los diagnósticos de riesgo la infección e integridad de la piel afectada es una de las principales preocupaciones del profesional y supone uno de sus mayores retos. Las múltiples punciones incrementan el riesgo de infección, requiriendo que el personal de enfermería esté atento a garantizar una larga vida útil del acceso vascular y de esta forma disminuir el riesgo de complicaciones. Pues después de las complicaciones renales, las infecciones constituyen la segunda razón principal de hospitalización en estos pacientes. Esto se explica por el hecho que son pacientes inmunocomprometidos y expuestos a procedimientos invasivos lo que los hace más susceptibles a las infecciones ${ }^{12,33,39}$.

De esta manera, enfermería utiliza todos los recursos disponibles para convertirse en una aliada del paciente y su familia y así brindar la educación, el apoyo y la orientación necesaria para que el régimen terapéutico sea efectivo y el paciente reciba los beneficios de un adecuado cuidado ${ }^{38}$.

\section{Educación del paciente en hemodiálisis}

La educación ofrece a los pacientes con Enfermedad Renal Crónica las bases de un conocimiento que facilita su participación activa en las decisiones sobre su propio cuidado para lograr los resultados deseados. Los objetivos educativos deben ser orientados a las necesidades del paciente, con un plan educativo que dé cuenta del estilo de aprendizaje del paciente y los posibles obstáculos de su proceso educativo. Al educar continuamente a los pacientes sobre los temas que son importantes para ellos, las enfermeras pueden ayudarlos a mejorar los resultados, crear actitudes positivas en cuanto a su tratamiento, y ser más independientes ${ }^{40}$.

\section{Autocuidado del paciente en hemodiálisis}

El paciente con insuficiencia renal se somete a cambios en su vida cotidiana, y el conocimiento de la hemodiálisis es crítico para que el paciente entienda las complicaciones, restricciones en alimentos, líquidos, uso de medicamentos, amenaza de su propia imagen, que generan estrés, conflicto y ansiedad, lo que interfiere con la adherencia a la terapia. Todos estos conocimientos interfieren con el auto-cuidado, dado que el individuo es el principal responsable de su calidad de vida, vivir con diálisis. Los efectos beneficiosos de la intervención de cuidados de enfermería, promueve un cambio positivo significativo en el autocuidado en comportamiento tales como la dieta, la presión arterial, control del peso, ejercicio y descanso, que los pacientes evidencian difíciles de controlar. Por lo tanto, el cuidado en salud de enfermería se debe utilizar activamente en la práctica clínica para que los pacientes de diálisis realicen autocuidado a través de la retroalimentación continua ${ }^{41-43}$.

\section{Importancia de la familia en el cuidado del paciente}

La familia en el paciente con insuficiencia renal es considerada parte esencial en la atención del cuidado y es importante en este proceso para generar prácticas de buenos hábitos que ayuden al paciente en su estilo de vida, tiene el papel de alentar y disminuir las tensiones que se vayan presentando a lo largo del proceso de la enfermedad, por esta razón el personal de enfermería debe apoyarse en la familia para la planificación de cuidados con el fin de generar beneficios al paciente. Para esto se debe analizar las condiciones físicas, económicas y socioculturales para conocer si realmente existen los medios en casa para brindar al enfermo la atención adecuada, ya que se deberá afrontar la enfermedad y también múltiples cambios en estilos de vida del paciente ${ }^{41}$.

Así mismo, es importante que se involucre a la familia, por su papel de apoyo en el tratamiento del paciente. EI apoyo y cooperación de enfermería hacia la familia, le ayuda a disminuir su incertidumbre y a prepararse para desempeñar un papel activo en el cuidado del paciente en el hogar, permitiéndole elegir el tipo de soporte más adecuado para las necesidades propias y decidir hasta donde desea tener parte en el cuidado de la persona enferma $35,38,39,41,42$.

Es por esta razón que el apoyo de la enfermera debe expresarse en la familia con diagnósticos enfocados 
en la familia para ayudar al paciente en hemodiálisis, que harán posible la búsqueda para diferentes tipos de apoyo en la planificación de cuidados que puedan generar diagnósticos positivos al paciente $\mathrm{e}^{41,42}$.

\section{Satisfacción del paciente como objetivo de calidad}

La evaluación de la satisfacción de los pacientes permite por un lado identificar aquellas áreas deficitarias desde el punto de vista del paciente y por otra parte permite evaluar los resultados de los cuidados, ya que las necesidades del paciente son el eje sobre el que se articulan las prestaciones asistenciales. Los aspectos que influyen en la satisfacción de los pacientes son "la puntualidad de las sesiones de hemodiálisis", "la rapidez con que consigue lo que necesita", "el interés del personal de enfermería por los pacientes" y el "tiempo de espera para ser atendido por el médico". En conclusión, el análisis de la satisfacción de los pacientes en hemodiálisis es muy útil para diseñar estrategias de mejora, sobre todo en aquellos aspectos dependientes del personal de Enfermería ${ }^{44}$.

Otro punto importante que se debe contemplar al atender al paciente es la necesidad de respetar sus deseos, tanto en la elección de tratamiento como en la posibilidad de modificar o retirarse de éste, por lo que un documento de voluntad anticipada es muy limitado en las unidades de diálisis, para establecer la anticipación del cuidado en donde se exprese una posición clara con respecto a la instauración de cuidados agresivos como el requerimiento de ventilación asistida, estado vegetativo, maniobras de resucitación en caso de parada cardiorrespiratoria en situaciones límite del fin de la vida, es importante la recogida y custodia de datos relativos a la voluntad ultima de los pacientes del respeto a sus decisiones, en caso de pérdida de su autonomía con esto muchas situaciones que se viven en la práctica clínica se podrían simplificar u optimizar a la voluntad del paciente ${ }^{43}$.

\section{Discusión}

Gracias a la creación de los modelos y teorías aplicadas a la enfermería se pudo definir el cuidado y establecer parámetros de partida para intervenir a un paciente. Los modelos y teorías guían el proceso general para elaborar de forma sistemática un método de trabajo de enfermería o Proceso de Atención de Enfermería (PAE). Más allá de la ejecución de tareas o la realización de procedimientos, el objetivo del desarrollo del conocimiento en enfermería es contribuir al acervo de conocimiento que se requiere para la práctica clínica en una gran variedad de situaciones, comprender las necesidades de las personas y aprender a cómo cuidar mejor de ellas ${ }^{45}$.

Algunos de los modelos y teorías aplicados a la atención de los pacientes en hemodiálisis descritos en los estudios revisados, fueron: el modelo de adaptación de Roy, la teoría de Autocuidado de Dorothea Orem y el modelo de las catorce necesidades básicas de Virginia Henderson. A pesar de la importancia que tiene para el desarrollo de la profesión la aplicación de modelos y teorías a la práctica clínica de enfermería, y de la evidencia en la mejora de cuidados de los pacientes, se observa que en el contexto del cuidado del paciente en hemodiálisis su uso es limitado y casi nulo.

Al mismo tiempo, se observa en los diferentes estudios la utilización frecuente de diagnósticos de enfermería según taxonomía NANDA, estableciéndose una serie de diagnósticos frecuentes, lo cual evidencia la importancia de emplear un lenguaje enfermero unificado, esto permite identificar problemas comunes de estos pacientes en diferentes contextos. Sin embargo se encuentran limitaciones a la hora de reflexionar y analizar sobre las intervenciones de enfermería, los resultados obtenidos de dichas intervenciones y su respectiva evaluación, puesto que los diferentes estudios han publicado solo las dos primeras fases del proceso de atención enfermero: valoración y diagnóstico, siendo indispensable conocer todas las fases del proceso y su impacto en la salud de los individuos y sus familias.

Por otra parte, se encuentran dentro de los elementos clave del cuidado del paciente en hemodiálisis, la educación del paciente, la participación del paciente en la planificación de sus cuidados, el autocuidado, la participación de la familia y las redes de apoyo, la satisfacción del paciente como objetivo de calidad y la importancia de las relaciones de la enfermera en cada una de las etapas de cuidado con el paciente y su familia. Estos elementos como pilares en la planificación de cuidados enfermeros, tienen que estar organizados dentro de una estructura de conocimiento, bases científicas y evidencia práctica dentro de un lenguaje estandarizado, con flexibilidad de aplicación de acuerdo a los diferentes contextos y momentos. 


\section{Conclusiones y recomendaciones}

La atención debe ser personalizada, enfocada al bienestar común, brindada por un profesional de enfermería (Recomendación B).

Los modelos y teorías de enfermería brindan posibilidades de intervención, de acción inmediata, e igualmente brinda al paciente las suficientes herramientas de cuidado. Sin embargo su uso es limitado en la práctica clínica diaria (Recomendación B).

Los fundamentos filosóficos y teorías de enfermería en la práctica clínica permite una visión más amplia, profunda y abarcadora de la enfermería que viabiliza el camino a la excelencia en la atención al paciente (Recomendación B).

La importancia de determinar los diagnósticos de enfermería comunes en los sujetos sometidos a hemodiálisis, tiene el propósito de ayudar a los profesionales de enfermería en la atención a los pacientes renales crónicos proporcionando herramientas para la planificación de la asistencia (Recomendación B).

La taxonomía NANDA permite el uso de lenguaje diagnósticos que son estandarizados para documentar lo realizado por enfermería y de una forma efectiva lograr un entendimiento interdisciplinar que se integre a la práctica asistencial. Para lograr adquirir este leguaje y conocimiento se requiere una formación continuada y sistemática desde las instituciones que forman a los profesionales en enfermería para el desarrollo de estas habilidades y de la adherencia a un modelo (Recomendación B).

La identificación de los diagnósticos de enfermería en pacientes con Enfermedad Renal Crónica en hemodiálisis, permite la planificación oportuna de las intervenciones y actividades de la profesión con un contenido científico en pro del bienestar del paciente (Recomendación B).

La educación continua de los pacientes sobre los temas que son importantes para ellos, permite a las enfermeras ayudarlos a mejorar los resultados, crear actitudes positivas en cuanto a su tratamiento, y ser más independientes (Recomendación B).

Los efectos beneficiosos de la intervención de cuidados de enfermería, promueve un cambio positivo significativo en el autocuidado (Recomendación B).
La familia en el paciente con insuficiencia renal es considerada parte esencial en la atención del cuidado y es importante en este proceso para generar prácticas de buenos hábitos. El personal de enfermería debe apoyarse en la familia para la planificación de cuidados con el fin de generar beneficios al paciente (Recomendación B).

El estudio de la satisfacción de los pacientes en hemodiálisis es útil para planificar estrategias de mejo$\mathrm{ra}$, sobre todo en aquellos aspectos dependientes del personal de Enfermería (Recomendación B).

\section{Recibido: 26 junio 15}

Revisado: 14 julio 15

Modificado: 20 enero 16

Aceptado: 27 febrero 16

\section{Bibliografía}

1. Acuña L, Soler L, Sánchez P, Martínez D, Torres $L$, Mercado $E$, et al. Situación de la enfermedad renal crónica en Colombia Fondo Colombiano de Enfermedades de Alto Costo2012. Disponible en: http://www.cuentadealtocosto.org/byblos/Docs/ Libro\%20ERC\%202012.pdf.

2. Ministerio de Salud y Protección Social, Ministerio de Hacienda y Crédito Público. Cuenta de Alto Costo 2013. Disponible en: http://www. cuentadealtocosto.org/.

3. Pinto N. Comprender la situación de cronicidad que viven nuestros pacientes. En: Universidad Nacional de Colombia. La investigación y el cuidado en América Latina. Colombia: Unibiblos, editor; 2005: 303-22.

4. Avellaneda A, Izquierdo M, Torrent F, Ramón J. Enfermedades raras: enfermedades crónicas que requierenunnuevoenfoquesociosanitario.AnSistema Sanit Navarra [Internet]. 2007;30(2). Disponible en: http://scielo.isciii.es/scielo.php?pid=S1137 $-66272007000300002 \&$ script=sci_arttext\&tlng=pt

5. Chaparro L. Trascender en un "vínculo especial" de cuidado: el paso de lo evidente a lo intangible. 
Bogotá, Colombia: Universidad Nacional de Colombia; 2009.

6. Moorhead S, Johnson M, Maas M, Swanson E. Clasificación de Resultados de Enfemería: desarrollo, perfeccionamiento y uso en la práctica, investigación y enseñanza. Cuarta ed. España: Elsevier Mosby; 2009.

7. Díaz W, Nieto C. Utilización del proceso de enfermería en el ejercicio clínico profesional en seis instituciones prestadoras de sevicios de salud en Santafé de Bogotá. Rev Salud Pública. 2003;14 (5):300-5.

8. Pons $A$, Escoda $T$, Brull $L$, Anento $S$, Martínez $E$, Porcar E. Proceso de atención de enfermería a un paciente con insuficiencia renal y hematuria 2005.

9. Abril M, Iglesias $R$, Jerez $A$, López $M$, Mañé $N$, Yuste E. Aplicación de una técnica cualitativa para la elaboración de un plan de atención al paciente pluripatológico en hemodiálisis. Rev Soc Esp Enferm Nefrol. 2004; 7 (4).

10. Luis M. Los diagnósticos enfermeros. Revisión crítica y guía práctica. Tercera ed. Barcelona: Masson; 2005

11. Hernangómez N. Plan de cuidados individualizado de un paciente con IRC en tratamiento con hemodiálisis. Reduca (Enfermería, Fisioterapia y Podología) Serie Trabajos de Fin de Grado. 2012;4 (1):127-94.

12. De Brito Poveda V, Da Silva Alves J, De Freitas Santos E, Garcia A. Diagnósticos de enfermería en pacientes sometidos a hemodiálisis. Enfermería global. 2014;13 (2).

13. Marcos S, Arenas M. Análisis de frecuencia de diagnósticos de enfermería para ser aplicados en planes de cuidados estandarizados a pacientes en diálisis. Encuesta realizada en el III Congreso de la Sociedad Latinoamericana de Enfermería Nefrológica2011. Disponible en: http://www. anbaweb.org/wp-content/uploads/2012/06/ Diagn \%C3\%B3sticos-de-Enfermer\%C3\%ADaen-Di\%C3\%Allisis.-SLANH-2012.pdf.

14. Marcos V. Plan de Cuidados Enfermeros en SM 2008.
15. García R, Vázquez M. Plan de cuidados al paciente oncológico y/o hematológico en la consulta de pruebas especiales del laboratorio de análisis clínicos. Rev Paraninfo Digital. 2010;10.

16. Cirera F, Ruiz M, Rosales V, J. M. Estandarización de cuidados del síndrome metabólico en diálisis peritoneal. Enferm Nefrol [revista en la Internet]. 2012;15 (2):129-37.

17. Elisângela F, Saraiva V, Marques E, Oliveira F, Mesquita $E$, Barbosa I. Implementation of nursing process to the patient submitted to hemodialysis. Journal Of Nursing. 2013.

18. Sackett D. Medicina basada en la evidencia: Cómo practicar y enseñar la MBE. En: Harcourt, editor. Madrid; 2011.

19. Gálvez A. La EBE como modelo de práctica: elementos conceptuales de la práctica basada en evidencias. In: INDEX F, editor. Enfermería Basada en la Evidencia Cómo incorporar la Investigación a la Práctica de los Cuidados. España 2007.

20. Guerra J, Muñoz $P$, Santos J. Las revisiones sistemáticas, niveles de evidencia y grados de recomendación Sevilla2003 [cited 2014]. Disponible en: http://www.guiamark.com/images/stories/mbe_ rev_sistematicas2.pdf.

21. CASPe. Critical Appraisal Skills Programme Español 2015. Disponible en: http://www.redcaspe.org/.

22. Von E, Altmanbd D, Eggera M, Pocockd S, Gotzschee P, Vandenbrouckef J. Declaración de la Iniciativa STROBE (Strengthening the Reporting of Observational studies in Epidemiology): directrices para la comunicación de estudios observacionales. Gac Sanit. 2008;22(2):144-50.

23. Rezende $R$, Porto $I$. Cuidado de enfermagem para clientela em hemodiálise: suas dimensões instrumentais e expressivas. Rev Eletr Enf [Internet]. 2009;11(2)(266-74).

24. Varez S, López M, Santos S, D. A. Perspectiva sobre modelos y teorías de enfermería en el ámbito de nefrologia. Rev Soc Esp Enferm Nefrol. 2008;11 (3).

25. Rodríguez M. Modelos de enfermería integrados al cuidado del paciente nefrológico en el contexto 
cubano. RevCubana Enfermer[revistaenlaInternet]. 2012;28(4):474-84.

26. Galache B. Diagnósticos de enfermería en pacientes con insuficiencia renal crónica en hemodiálisis. Rev Soc Esp Enferm Nefrol. 2004; 7(3):158-63.

27. FRAZÃO C. Diagnósticos de enfermagem em pacientes submetidos à hemodiálise: semelhanças entre 0 modelo de adaptação e a NANDA internacional. Dissertação (Mestrado em Assistência à Saúde) - Universidade Federal do Rio Grande do Norte, Natal, 20122012.

28. Frazão C, Medeiros A, F. LeS, Dantas J, Brandão A. Diagnósticos de enfermagem em pacientes renais crônicos em hemodiálise. Acta paul enferm. 2014;27( 1 ):40-3.

29. Frazão C, Fernandes $M$, Nunes $M$, Sá J, Lopes M, Lira A. Components of a Roy's adaptation model in patients undergoing hemodialysis. Rev Gaúcha Enferm. 2013;34(4):45-52.

30. Franco A, González E, Cano I, Domínguez I, de la Salud I, Salgado J, et al. Planes de cuidados enfermeros en SM 2008. Available from: http:// www.saludmentalextremadura.com/fileadmin/ documentos/Documentos_Tecnicos_SES/PLANES_ DE_CUIDADOS_ENFERMEROS_EN_SM.pdf.

31. FUDEN. Tipos de Diagnósticos de Enfermería. Observatorio de Metodología Enfermera Madrid, España [cited 2015]. Available from: http://www. ome.es/02_02_01_desa.cfm?id=109.

32. Braga L, Galdino J, da Silva Borba L, Brandão de Carvalho A. Diagnósticos de enfermagem em adultos em tratamento de hemodiálise. Rev Acta Paul Enferm. 2008;21(Número Especial):160-3.

33. Dallé J, de Fátima A. Diagnósticos de enfermagem identificados em pacientes hospitalizados durante sessões de hemodiálise. Rev Acta Paul Enferm. 2012;25(4):504-10.

34.Bisca M, Marques I. Perfil de diagnósticos de enfermería antes de iniciar el tratamiento hemodialitico. Revista Brasileira de Enfermagem. 2010;63(3):435-9.

35. dos Santos I, de Paula R, Miguéis L. Necessidades de orientação de enfermagem para 0 autocuidado de clientes em terapia de hemodiálise Rev Bras Enferm, Brasília. 2011;64(2):335-42.

36. Fernandes $M$, Medeiros $A$, Macedo B, Vitorino A, Lopes M, Lira A. Prevalence of nursing diagnosis of fluid volume excess in patients undergoing hemodialysis. Revista Da Escola de Enfermagem Da Usp. 2014;48(3):446-53.

37. Orem D. Modelo de Orem: La teoría de Enfermería. Una Teoría General. Una Teoría General. cap 3. Madrid: Masson- Salvat; 1993. p. 7-22.

38. Bisca M, Marques I. Profile of nursing diagnoses before starting hemodialitic treatment. Rev Bras Enferm. 2010.

39. Aparecida T, Lappann N. Providing and receiving nursing care during hemodialysis. Rev Acta paul enferm. 2009;22.

40. Wingard R. Patient Education and the Nursing Process: Meeting the Patient's Needs. Nephrology nursing journal: journal of the American Nephrology Nurses' Association. 2005;32(2):211-4.

41. Ziegert K, Fridlund B, Lidell E. Professional support for next of kin of patients receiving chronic haemodialysis treatment: a content analysis study of nursing documentation. Revista J Clin Nurs. 2007.

42. Mi-Kyoung C. Effect of health contract intervention on renal dialysis patients in Korea. Rev Nursing \& Health Sciences. 2013;15(1):86-93.

43. Sánchez J, Rodríguez A, Baldan S, Cigarrán S, Herrero J, Maduel F, et al. Evaluación de la opinión de los pacientes con enfermedad renal crónica en diálisis respecto al fin de la vida y la planificación anticipada de cuidados. Revista Nefrología. 2011;31(4):449-56.

44. Hernández Meca $M^{a}$ Encarnación OGA, Mora Canales Javier, Lorenzo Martínez Susana, López Revuelta Katia. Satisfacción del paciente en una unidad de hemodiálisis: Objetivo de calidad asistencial en enfermería. Rev Soc Esp Enferm Nefrol [revista en la Internet]. 2005;8 (2).

45. De la Cuesta C. La Investigación Cualitativa y el Desarrollo del Conocimiento en Enfermería. Alicante 2010. p. 762-6. 